\title{
EXTENDING LIAPUNOV'S SECOND METHOD TO NON-LIPSCHITZ LIAPUNOV FUNCTIONS
}

\author{
BY JAMES A. YORKE ${ }^{1}$
}

Communicated by H. A. Antosiewicz, October 3, 1967

The behavior of solutions of an ordinary differential equation (E)

$$
d x / d t=f(t, x),
$$

where $f: U \rightarrow R^{n}$ is continuous on the open set $U \subset R \times R^{n}$, is frequently studied by means of a continuous function $V: U \rightarrow R$. It is sometimes unnecessary to know the solutions explicitly. If for example $V$ is independent of $t, V\left(x_{0}\right)=0$ for some $x_{0}, V(x)>0$ for $x \neq x_{0}$, and if for each solution $\phi$ of $(\mathrm{E}), V(\phi(t))$ is a monotonically decreasing function of $t$ for $t \geqq 0$, then $x_{0}$ is a stable critical point of (E). For $V$ a $C^{1}$ function, Liapunov defined

$$
\dot{V}(t, x)=\frac{\partial}{\partial t} V(t, x)+\left\langle\operatorname{grad}_{x} V(t, x), f(t, x)\right\rangle
$$

where $\langle\cdot, \cdot\rangle$ denotes the inner product in $R^{n}$. He observed that for any solution $\phi$,

$$
\dot{V}(t, \phi(t))=d V(t, \phi(t)) / d t
$$

hence the rate of change of $V(t, \phi(t))$ can be calculated directly from $V$ and $f$ without knowing the solutions when $V$ is a $C^{1}$ function. Sometimes a likely function $V$ is not $C^{1}$, and for converse theorems frequently the most difficult problem is proving $V$ can be chosen to be a smooth function. A theory was thus developed for $V \in C^{0}$ ( $V$ locally Lipschitz in $x$ ) primarily by Yoshizawa [2, p. 4] with earlier results by Okamura [1]. We will mean by $\phi\left(\cdot ; t_{0}, x_{0}\right)$ that $\phi$ is a solution of (E) such that $\phi\left(t_{0}\right)=x_{0}$. When we refer to the domain of $\phi$, we will assume that $\phi$ cannot be extended to a larger domain and still be a solution. Define for a solution $\phi=\phi(\cdot ; t, x)$

$$
\begin{aligned}
& V^{\prime}(t, x)=\liminf _{\tau \rightarrow+0} \tau^{-1}[V(t+\tau, \phi(t+\tau))-V(t, \phi(t))], \\
& \dot{V}(t, x)=\liminf _{\tau \rightarrow+0} \tau^{-1}[V(t+\tau, x+\tau f(t, x))-V(t, x)] .
\end{aligned}
$$

In (1) and (2) we use the so-called lower right-hand Dini derivate. For $V \in C^{0}$, if $W$ is a continuous real-valued function and $\dot{V}(t, x)$

I Partially supported by National Science Foundation grant GP 6114. 
$\leqq W(t, x)$ for one of the Dini derivates, then the inequality will hold for all four Dini derivates so it makes no difference which Dini derivate is used. In our development "lim inf, $\tau \rightarrow+0$ " is better than any other derivate.

If $V$ is $C^{0}$, then $V^{\prime}(t, x)$ does not depend on the particular solution chosen and in fact $V^{\prime}(t, x)=\dot{V}(t, x)$. For $V$ only a continuous function, $V^{\prime}(t, x)$ does depend on the particular solution through $(t, x)$ so when we write $V^{\prime}(t, \phi(t))$, it will be implicit that $V^{\prime}$ is evaluated with respect to $\phi$. We have found examples with the following behaviors when $V$ is only continuous:

(1) $\dot{V} \equiv 0$ on $U$ and yet there exists a solution $\phi$ such that $V^{\prime}(t, \phi(t))$ $\equiv 1$ for $t \in R$ (or we may have $V^{\prime}(t, \phi(t)) \equiv-1$ for $t \in R$ ).

(2) $\dot{V} \equiv 0$ on $U$ and yet there exists a point $x_{0}$ such that for every solution $\phi$ of (E) with $\phi(0)=x_{0}$, we have $V^{\prime}(t, \phi(t))=1$ for all $t \leqq 0$ (or we may have $=-1$ for all $t \leqq 0)$.

(3) Solutions of (E) unique and $V^{\prime}(t, \phi(t)) \leqq 0$ for all $t$ and all solutions $\phi$ and yet there exists a solution $\phi$ such that $\dot{V}(t, \phi(t))=1$ for all $t \in R$.

Because of the above examples, Theorem 1, which is apparently new, is perhaps the best result that can be obtained when $V$ is continuous but not necessarily $C^{0}$.

THEOREM 1. If $W: U \rightarrow R$ is continuous and $\dot{V}(t, x) \leqq W(t, x)$ for all $(t, x) \in U$, then for each $\left(t_{0}, x_{0}\right) \in U$ there exists a solution $\phi=\phi\left(\cdot ; t_{0}, x_{0}\right)$, such that for all $t \geqq t_{0}$ in the domain of $\phi$,

$$
V^{\prime}(t, \phi(t)) \leqq W(t, \phi(t)) .
$$

One main purpose of this note, however, is to introduce another derivate $V^{*}$ of $V$ which allows nearly the entire Liapunov theory of $C^{0}$ Liapunov functions to hold for $V$ which need only be continuous. Let $|y|$ denote the norm of $y \in R^{n}$. Define

$$
\stackrel{*}{V}(t, x)=\liminf _{\tau \rightarrow+0 ;|y| \rightarrow 0} \tau^{-1}[V(t+\tau, x+\tau y+\tau f(t, x))-V(t, x)] .
$$

From the definition it follows that $\stackrel{*}{V} \leqq \dot{V}$ and $\stackrel{*}{V} \leqq V^{\prime}$ (calculated along any solution). We can prove Theorem 1 is true with $\dot{V}$ replaced by $\stackrel{*}{V}$. From this and $\stackrel{*}{V} \leqq V^{\prime}$ we have

Theorem 2. Let $W: U \rightarrow R$ be continuous. Then the following two conditions are equivalent.

(2.1) For all $\left(t_{0}, x_{0}\right) \in U$, there exists a solution $\phi=\phi\left(\cdot ; t_{0}, x_{0}\right)$ such that $V^{\prime}(t, \phi(t)) \leqq W(t, \phi(t))$ for $t \geqq t_{0}$ in the domain of $\phi$. 


$$
\stackrel{*}{V}(t, x) \leqq W(t, x) \text { for all }(t, x) \in U .
$$

CoRollary. If solutions are unique and $V: U \rightarrow R$ is continuous, $V(t, x) \leqq 0$ for all $(t, x) \in U$ iff for every solution $\phi, V(t, \phi(t))$ is a monotonically decreasing function for $t$ in the domain of $\phi$.

Theorem 1 follows immediately from Theorem 2, and it is not difficult to derive the corollary from Theorem 2 .

Sketch of Proof of Theorem 2. For any $\left(t_{0}, x_{0}\right) \in U$ and $\epsilon>0$ we may choose $\tau, y$ such that $0<\tau<\epsilon,|y|<\epsilon$, and the line segment between $\left(t_{0}, x_{0}\right)$ and $\left(t_{1}, x_{1}\right)=\left(t_{0}+\tau, x_{0}+\tau y+\tau f\left(t_{0}, x_{0}\right)\right)$ lies in $U$ and

$$
\tau^{-1}\left[V\left(t_{0}+\tau, x_{0}+\tau y+\tau f\left(t_{0}, x_{0}\right)\right)-V\left(t_{0}, x_{0}\right)\right]-W\left(t_{0}, x_{0}\right)<\epsilon .
$$

By a sequence of such choices, $\left(t_{0}, x_{0}\right),\left(t_{1}, x_{1}\right), \cdots,\left(t_{i}, x_{i}\right), \cdots$, we may construct a piecewise linear "approximate" solution. The sequence $\left\{\left(t_{i}, x_{i}\right)\right\}$ can be chosen so as not to have a cluster point in $U$. When the approximate solutions are properly chosen, some subsequence converges to a solution of (E) satisfying $V^{\prime}(t, \phi(t)) \leqq W(t, \phi(t))$ for $t \geqq t_{0}$.

APPLICATION. The use of $\stackrel{*}{V}$ rather than $\dot{V}$ will of ten allow better theorems with simpler proofs, particularly for converse theorems, as in the following application.

We assume now that for some $\eta>0$ the set $D_{\eta}=\{(t, x):|x| \leqq \eta$ and $t \geqq 0\} \subset U$. Following Strauss [3], we assume solutions of (E) are unique and we define

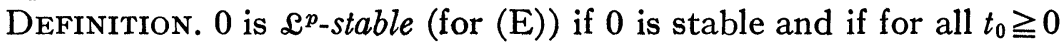
there exists a $\delta=\delta\left(t_{0}\right)>0$ such that

$$
\int_{t_{0}}^{\infty}\left|\phi\left(t ; t_{0}, x_{0}\right)\right|^{p} d t<\infty \text { for all }\left|x_{0}\right|<\delta .
$$

THEOREM 3. For any $p>0$ the following are equivalent.

(3.1) There exists $\rho, \eta>\rho>0$, and a continuous positive definite function $V: D_{\rho} \rightarrow[0, \infty)$ such that $V(t, 0) \equiv 0$ and for some $c>0$

$$
\stackrel{*}{V}(t, x) \leqq c|x|^{p} \quad \text { for }(t, x) \in D_{\rho} \text {. }
$$

(3.2) 0 is $\mathfrak{L}^{p}$-stable.

Theorem 3 is essentially due to Strauss [3] except that he did not have available Theorems 1 and 2 . He showed that if $V \in C^{0}$, then (3.1) implies (3.2), and to conclude (3.1) and $V \in C^{0}$ he had to assume (3.2) and $f \in C^{1}$ and

(3.3) For some $\rho$ there exists a function $\psi \in \mathcal{L}^{p}$ on $[0, \infty)$ such that 


$$
\left|\theta\left(t+t_{0}, t_{0}, x_{0}\right)\right| \leqq \psi(t), \quad \text { for }\left(t_{0}, x_{0}\right) \in D_{\rho}, \quad t \geqq 0,
$$

where $\theta$ is the matrix of partial derivatives $\phi_{x_{0}}\left(t ; t_{0}, x_{0}\right)$.

\section{BIBLIOGRAPHY}

1. H. Okamura, Condition nécessaire et suffisante remplie par les équations différentielles ordinaires sans points de Peano, Mem. Colloq. Sci. Kyoto Imperial Univ. Ser. A 24 (1942), 21-28.

2. Taro Yoshizawa, Stability theory by Liapunov's second method, Math. Soc. of Japan, Tokyo, 1966.

3. Aaron Strauss, Liapunov functions and $L^{p}$ solutions of differential equations, Trans. Amer. Math. Soc. 119 (1965), 37-50.

Institute for Fluid Dynamics and Applied Mathematics, University of MARYLAND 